\title{
EMBARAZO EN EDAD MATERNA AVANZADA: RESULTADOS PERINATALES
}

\author{
Dasio Ayala Moreno ${ }^{1}$, Antonio Mambret Luna Figueroa², Félix Dasio Ayala Peralta ${ }^{3}$, Enrique Guevara Ríos ${ }^{4}$, \\ César Carranza Asmat ${ }^{5}$, Emma Ysabel Quiñones Pereyra ${ }^{6}$, Richard Ayala Palomino ${ }^{7}$, \\ Luis Alberto Quiñones Vásquez ${ }^{8}$.
}

\begin{abstract}
RESUMEN
Objetivo: Identificar los resultados perinatales en gestantes con edad materna avanzada (EMA) atendidas en el Instituto Nacional Materno Perinatal. Materiales y métodos: Estudio observacional, descriptivo y retrospectivo de 321 egresos maternos ocurridas durante el periodo de 1 de enero al 30 de junio 2016. Resultados: Edad promedio 38,8 $\pm 3,4$ años. Conviviente en $63,9 \%$, secundaria completa $68,3 \%$ y ama de casa $80,4 \%$. Los principales hallazgos perinatales fueron: recién nacidos (RN) con bajo peso al nacer (15\%); presencia de líquido amniótico meconial(17\%); circular de cordón nucal fetal (18\%); macrosomía fetal (10\%) y malformación congénita en 3,7\% principalmente Sindrome de Down. La tasa de mortalidad fetal (TMF) arroja la cifra de 2,55 por mil nacimientos/año. Conclusión: Los hallazgos perinatales en EMA fueron RN con bajo peso al nacer, líquido amniótico meconial, distocia funicular, macrosomía fetal y malformación congénita principalmente Sindrome de Down.
\end{abstract}

Palabras clave: Edad materna avanzada; Hallazgos perinatales; Embarazo de alto riesgo (Fuente DeCS BIREME).

\section{PREGNANCY IN ADVANCED MATERNAL AGE: PERINATAL RESULTS}

\begin{abstract}
Objective. To identify perinatal outcomes in pregnant women with advanced maternal age (EMA) treated at the National Maternal Perinatal Institute. Materials and methods. Observational, descriptive and retrospective study of 321 maternal outgoings occurred during the period from January 1 to June 30, 2016. Results. Average age $38.8 \pm 3.4$ years. Living in $63.9 \%$, high school $68.3 \%$ and housewife $80.4 \%$. The main perinatal findings were: Newborns (RN) with low birth weight (15\%); Presence of meconium amniotic fluid (17\%); Circular fetal nuchal cord (18\%); Fetal macrosomia (10\%) and congenital malformation in 3.7\%, mainly Down's Syndrome. The fetal mortality rate (FMR) shows the figure of 2.55 per thousand births / year. Conclusion. The perinatal findings in EMA were Newborns with low birth weight, meconium amniotic fluid, funicular dystocia, fetal macrosomia and congenital malformation, mainly Down's Syndrome.
\end{abstract}

Key words: Advanced maternal age; Perinatal findings; High risk pregnancy (Source: MeSH NLM).

\section{INTRODUCCIÓN}

La edad materna avanzada (EMA) es definida como aquella mujer que se embaraza a partir de $\geq 35$ años de edad ${ }^{1-3}$

La EMA trae aparejado complicaciones maternas, tales como: trastornos hipertensivos, diabetes gestacional, leiomiomatosis uterina, presentaciones anormales, hemorragia obstétrica y mayor índice de cesáreas; así como, complicaciones perinatales como: restricción del crecimiento intrauterino, parto pretérmino, bajo peso al nacer, puntuación baja en la escala de Apgar, incremento de anomalías congénitas, aborto; los cuales conllevan al incremento de morbi-mortalidad materno perinatal ${ }^{4,5-13}$

En el Instituto Nacional Materno perinata ${ }^{14}$ durante el periodo 2016 ocurrieron 21,983 nacimientos/año, de los cuales corresponden a edad materna avanzada $\geq 35$ años de edad con 4,300 gestantes/año que representan el $19,56 \% ; y \geq 40$ años de edad con 1222 gestantes/año que representan el $4,89 \%$.

El objetivo de la presente investigación es identificar los resultados perinatales en gestantes con edad materna

\footnotetext{
Médico Cirujano. Universidad Nacional Federico Villarreal, Lima-Perú.

Médico GínecoObstetra. Profesor Principal de la Universidad Nacional Mayor de San Marcos. Director Ejecutivo de Investigación, Docencia y Atención en Obstetricia y Ginecología del Instituto Nacional Materno Perinatal. Lima-Perú.

3 Médico GínecoObstetra. Magíster en Salud Reproductiva. Profesor de la Facultad de Medicina Humana de la Universidad San Martín de Porres y de Ciencias de la Salud de la Universidad Norbert Wiener. Jefe del Servicio de Climaterio y Menopausia del Instituto Nacional Materno Perinatal. Miembro de la Red Latinoamericana de Investigadores en Climaterio (REDLINC). Lima-Perú.

4 Médico GínecoObstetra. Director del Instituto Nacional Materno Perinatal. Miembro de American College of Obstetricians and Gynecologists (ACOG). Coordinador de Asistencia Técnica del Instituto de Salud Popular, Consultor de Pathfinder International. Docente de la Universidad Nacional Mayor de San Marcos, Lima, Perú.

5 Médico GínecoObstetra. Profesor Asociado de la Universidad Nacional Mayor de San Marcos. Director Ejecutivo de la Oficina de Investigación y Docencia Especializada. Instituto Nacional Materno Perinatal. Lima-Perú.

- Médico Cirujano. Universidad San Martín de Porres. Lima-Perú.

Médico Cirujano. Universidad Privada San Juan Bautista. Lima-Perú

Médico GínecoObstetra. Asistente del Servicio de Obstetricia C. Instituto Nacional Materno Perinatal. Lima-Perú.
} 
avanzada atendidas en el Instituto Nacional Materno Perinatal durante el periodo comprendido desde 1 de enero al 30 de junio 2016.

\section{MATERIALES Y MÉTODOS}

Investigación observacional, descriptivo y retrospectivo.

Población: Gestantes $\geq 35$ años con edad, nulípara o multípara, que fueron atendidas en INMP, desde 1 de enero al 30 de junio del 2016.

Muestra: El número total de nacimientos durante el periodo 2016 en el Instituto Nacional Materno Perinatal fueron 21983 y el número total de egresos maternos en edad materna avanzada fueron 4300/año. El cálculo del tamaño de la muestra con grado de confianza al 95\% y error $5 \%$ corresponden 321 casos estudiados que fueron seleccionadas utilizando el muestreo aleatorio simple.

\section{Criterios de inclusión}

- Historia clínica de gestante con edad materna avanzada $\geq 35$ años de edad.

- Historia clínica de gestantes con edad gestacional confiable por clínica ó ultrasonografía $\geq 22$ semanas.

- Historia clínica de gestantes que culminaron en parto vaginal o cesárea.

- Historias clínicas de egresos maternos registrados en el Instituto Nacional Materno Perinatal durante el periodo de 01 de enero al 30 de junio 2016

\section{Criterios de exclusión}

- Todas las pacientes que no cumplan los criterios de inclusión.

- Gestantes menores de 35 años de edad.

- Gestantes atendidas fuera del Instituto.

Técnicas de recolección de datos: Los datos fueron obtenidos a partir de las historias clínicas de las gestantes atendidas en el Departamento de Obstetricia y Perinatología del INMP cuyo egreso materno corresponde al primer semestre 2016, que fueron consignados en la ficha de recolección de datos, elaborada para dicho propósito.

Técnicas para el procesamiento de la información: Se elaboró una base de datos en el programa Microsoft Windows Excel. Para el procesamiento de la información se utilizó el paquete estadístico SPSS - PASW versión 20 (Stadistical Packaged for the Social Sciences - Predictive Analytics Software) para la elaboración de tablas descriptivas, de frecuencia, así como también de gráficos.

Aspectos éticos: Todos los procedimientos del presente estudio tratan de preservar la integridad y los derechos fundamentales de los pacientes sujetos a investigación, de acuerdo con los lineamientos de las buenas prácticas clínicas y de ética en investigación biomédica. Se garantizó la confidencialidad de los datos obtenidos.

\section{RESULTADOS}

Tabla 1. Características sociodemográficas en edad materna avanzada. Instituto Nacional Materno Perinatal, enero-junio 2016.

\begin{tabular}{|c|c|c|}
\hline $\begin{array}{c}\text { Características } \\
\text { Sociodemográficas }\end{array}$ & \multicolumn{2}{|c|}{$X \pm$ DS (min. - máx.) } \\
\hline \multirow{2}{*}{ Edad } & \multicolumn{2}{|c|}{$38,8 \pm 3,4(35-47)$} \\
\hline & $\mathbf{N}$ & $\%$ \\
\hline 35 - 39 años & 194 & $60,4 \%$ \\
\hline 40 - 44 años & 106 & $33,0 \%$ \\
\hline $45-49$ años & 21 & $6,6 \%$ \\
\hline \multicolumn{3}{|l|}{ Estado Civil } \\
\hline Soltera & 25 & $7,8 \%$ \\
\hline Conviviente & 205 & $63,9 \%$ \\
\hline Casada & 91 & $28,3 \%$ \\
\hline \multicolumn{3}{|l|}{ Escolaridad } \\
\hline Sin instrucción & 1 & $0,3 \%$ \\
\hline Primaria & 36 & $11,3 \%$ \\
\hline Secundaria completa & 218 & $68,3 \%$ \\
\hline Superior técnico & 35 & $11,0 \%$ \\
\hline Superior universitario & 29 & $9,1 \%$ \\
\hline \multicolumn{3}{|l|}{ Ocupación } \\
\hline Ama de casa & 255 & $80,4 \%$ \\
\hline Empleada & 29 & $9,1 \%$ \\
\hline Obrera & 7 & $2,2 \%$ \\
\hline Independiente & 26 & $8,2 \%$ \\
\hline TOTAL (n) & 321 & $100,00 \%$ \\
\hline
\end{tabular}

En la tabla 1 se aprecia que la edad promedio de las gestantes es 38,8 $\pm 3,4$ años, donde la mayoría pertenece al grupo etario de 35 a 39 años con $60,4 \%$; estado conyugal convivientes en $63,9 \%$; secundaria completa el 68,3 ; y ama de casa $80,4 \%$.

En la tabla 2 se aprecia que predomina la multiparidad seguida de primíparas en $17 \%$ de casos .

En relación a la edad gestacional, se aprecia en la tabla 3 que corresponden a pretérmino $\leq 36$ semanas en $22,0 \%$ (67 casos) que es un factor de riesgo importante en esta etapa de la vida.

Tabla 2. Paridad según edad materna avanzada. Instituto Nacional Materno Perinatal. Enero-junio 2016.

\begin{tabular}{|c|c|c|c|c|c|c|c|c|}
\hline \multirow{3}{*}{$\begin{array}{c}\text { Edad } \\
\text { materna } \\
\text { (años) }\end{array}$} & \multicolumn{8}{|c|}{ Paridad } \\
\hline & \multicolumn{2}{|c|}{ Nulípara } & \multicolumn{2}{|c|}{ Primípara } & \multicolumn{2}{|c|}{ Multípara } & \multicolumn{2}{|c|}{$\begin{array}{c}\text { Gran } \\
\text { multípara } \geq 6\end{array}$} \\
\hline & $\mathbf{N}$ & $\%$ & $\mathbf{N}$ & $\%$ & $\mathbf{N}$ & $\%$ & $\mathbf{N}$ & $\%$ \\
\hline $35-39$ & 11 & $3,7 \%$ & 23 & $7,66 \%$ & 145 & $48,0 \%$ & 5 & $1,5 \%$ \\
\hline $40-44$ & 3 & $1,0 \%$ & 10 & $3,33 \%$ & 73 & $24,1 \%$ & 14 & $4,3 \%$ \\
\hline $45-49$ & 1 & $0,3 \%$ & 3 & $1,0 \%$ & 12 & $3,9 \%$ & 4 & $1,2 \%$ \\
\hline Total & 15 & $5,0 \%$ & 36 & $12,0 \%$ & 230 & $76,0 \%$ & 23 & $7,0 \%$ \\
\hline
\end{tabular}


Tabla 3. Edad gestacional según edad materna avanzada. Instituto Nacional Materno Perinatal. Enero-junio 2016.

\begin{tabular}{lcccccccc}
\hline Edad & \multicolumn{6}{c}{ EDAD GESTACIONAL (semanas) } \\
\cline { 2 - 9 } $\begin{array}{c}\text { materna } \\
\text { avanzada }\end{array}$ & $\mathbf{2 2}$ a $\mathbf{2 7}$ & $\mathbf{2 8}$ a $\mathbf{3 6}$ & $\mathbf{3 7}$ a $\mathbf{4 1}$ & $\mathbf{4 2}$ a más \\
\cline { 2 - 9 } (años) & $\mathbf{N}$ & $\%$ & $\mathbf{N}$ & $\mathbf{\%}$ & $\mathbf{N}$ & $\%$ & $\mathbf{N}$ & $\%$ \\
\hline $35-39$ & 5 & $2,0 \%$ & 31 & $10,0 \%$ & 151 & $\mathbf{4 8 , 7} \%$ & 2 & $0,7 \%$ \\
$40-44$ & 0 & $0,0 \%$ & 24 & $7,7 \%$ & 75 & $24,2 \%$ & 0 & $0,0 \%$ \\
$45-49$ & 0 & $0,0 \%$ & 7 & $2,3 \%$ & 13 & $4,1 \%$ & 1 & $0,3 \%$ \\
Total & 5 & $2,0 \%$ & 62 & $20,0 \%$ & 239 & $77,0 \%$ & 3 & $1,0 \%$ \\
\hline
\end{tabular}

Tabla 4. Vía de culminación del parto según edad materna avanzada. Instituto Nacional Materno Perinatal. Enerojunio 2016.

\begin{tabular}{|c|c|c|c|c|}
\hline \multirow{3}{*}{$\begin{array}{c}\text { Edad materna } \\
\text { avanzada }\end{array}$} & \multicolumn{4}{|c|}{ Tipo de parto } \\
\hline & \multicolumn{2}{|c|}{ Vaginal } & \multicolumn{2}{|c|}{ Cesárea } \\
\hline & $\mathbf{N}$ & $\%$ & $\mathbf{N}$ & $\%$ \\
\hline $35-39$ años & 87 & $27,1 \%$ & 109 & $33,9 \%$ \\
\hline 40 - 44 años & 44 & $13,8 \%$ & 60 & $18,7 \%$ \\
\hline 45 - 49 años & 10 & $3,1 \%$ & 11 & $3,4 \%$ \\
\hline Total & 141 & $44,0 \%$ & 180 & $56,0 \%$ \\
\hline
\end{tabular}

En la tabla 4 se aprecia que la vía de culminación del parto predominante fue la cesárea en el $56 \%$ de casos .

Tabla 5. Puntuación Apgar del recién nacido al primer minuto según edad materna avanzada. Instituto Nacional Materno Perinatal. Enero-junio 2016.

\begin{tabular}{|c|c|c|c|c|c|c|}
\hline \multirow{3}{*}{$\begin{array}{c}\text { Edad } \\
\text { materna } \\
\text { (años) }\end{array}$} & \multicolumn{6}{|c|}{$\begin{array}{c}\text { Puntuación Apgar del recién nacido } \\
\text { al primer minuto }\end{array}$} \\
\hline & \multicolumn{2}{|c|}{$1-3$} & \multicolumn{2}{|c|}{ 4-6 } & \multicolumn{2}{|c|}{$7-10$} \\
\hline & $\mathbf{N}$ & $\%$ & $\mathbf{N}$ & $\%$ & $\mathbf{N}$ & $\%$ \\
\hline $35-39$ & 9 & $3,0 \%$ & 11 & $3,4 \%$ & 172 & $54,1 \%$ \\
\hline $40-44$ & 8 & $2,7 \%$ & 5 & $1,6 \%$ & 91 & $28,6 \%$ \\
\hline $45-49$ & 1 & $0,3 \%$ & 0 & $0,0 \%$ & 20 & $6,3 \%$ \\
\hline Total & 18 & $6,0 \%$ & 16 & $5,0 \%$ & 283 & $89,0 \%$ \\
\hline
\end{tabular}

Como se aprecia en la tabla 5 , en relación a la puntuación de Apgar del recién nacido(RN) al primer minuto, se encontró Apgar bajo $<7$ en el $11 \%$ de la casuística y de ellos Apgar $<3$ en el $6 \%$. La mayoría de casos en $89 \%$ presentaron puntuación de Apgar normal.

Tabla 6. Puntuación Apgar del recién nacido a los 5 minutos según edad materna avanzada. Instituto Nacional Materno Perinatal. Enero-junio 2016

\begin{tabular}{|c|c|c|c|c|c|c|}
\hline \multirow{3}{*}{$\begin{array}{c}\text { Edad } \\
\text { materna } \\
\text { (Años) }\end{array}$} & \multicolumn{6}{|c|}{$\begin{array}{l}\text { Puntuación Apgar } \\
\text { del recién nacido a los } 5 \text { minutos }\end{array}$} \\
\hline & \multicolumn{2}{|r|}{$1-3$} & \multicolumn{2}{|r|}{ 4-6 } & \multicolumn{2}{|c|}{$7-10$} \\
\hline & $\mathbf{N}$ & $\%$ & $\mathbf{N}$ & $\%$ & $\mathbf{N}$ & $\%$ \\
\hline $35-39$ & 7 & $2,2 \%$ & 2 & $0,6 \%$ & 183 & $57,8 \%$ \\
\hline $40-44$ & 5 & $1,6 \%$ & 1 & $0,3 \%$ & 98 & $30,9 \%$ \\
\hline $45-49$ & 1 & $0,3 \%$ & 0 & $0,0 \%$ & 20 & $6,3 \%$ \\
\hline Total & 13 & $4,1 \%$ & 3 & $0,9 \%$ & 301 & $95,0 \%$ \\
\hline
\end{tabular}

En la tabla 6 se aprecia que la puntuación de Apgar del $\mathrm{RN}$ a los cinco (5) minutos, revela que presentó Apgar bajo $<7$ solo en $5 \%$ de la casos y de ellos Apgar $<3$ en solo $4,1 \%$.

Tabla 7. Peso del recién nacido según edad materna avanzada. Instituto Nacional Materno Perinatal. Enerojunio 2016.

\begin{tabular}{ccccccc}
\hline \multirow{2}{*}{$\begin{array}{c}\text { Edad } \\
\text { materna }\end{array}$} & \multicolumn{5}{c}{ Peso al nacer } \\
\cline { 2 - 7 } $\begin{array}{c}\text { avanzada } \\
\text { (Años) }\end{array}$ & $\mathbf{< 2 5 0 0 g}$ & $\mathbf{2 5 0 0 - 3 9 9 9 g}$ & $\begin{array}{c}\text { 4000 a más } \\
\text { gramos }\end{array}$ \\
\cline { 2 - 7 } & $\mathbf{N}$ & $\%$ & $\mathbf{N}$ & $\mathbf{\%}$ & $\mathbf{N}$ & $\%$ \\
\hline $35-39$ & 23 & $7,0 \%$ & 154 & $48,0 \%$ & 19 & $6,1 \%$ \\
\hline $40-44$ & 16 & $5,0 \%$ & 77 & $24,0 \%$ & 11 & $3,6 \%$ \\
$45-49$ & 9 & $3,0 \%$ & 11 & $3,0 \%$ & 1 & $0,3 \%$ \\
Total & $\mathbf{4 8}$ & $\mathbf{1 5 , 0 \%}$ & $\mathbf{2 4 2}$ & $\mathbf{7 5 , 0} \%$ & $\mathbf{3 1}$ & $\mathbf{1 0 , 0} \%$ \\
\hline
\end{tabular}

En relación al peso del RN, como se aprecia en la tabla 7, corresponden en el $15 \%$ a $\mathrm{RN}$ bajo peso al nacer (BPN) y de ellos más de la mitad con $8 \%$ corresponden al grupo etario de 40-49 años de edad. Por otro lado, se encontró macrosomía en el $10 \%$.

Tabla 8. Coloración del líquido amniótico según edad materna avanzada. Instituto Nacional Materno Perinatal. Enero-junio 2016.

\begin{tabular}{ccccccc}
\hline \multirow{2}{*}{$\begin{array}{c}\text { Edad } \\
\text { materna }\end{array}$} & \multicolumn{3}{c}{ Coloración del líquido amniótico } \\
\cline { 2 - 7 } avanzada & \multicolumn{2}{c}{ Claro } & \multicolumn{3}{c}{ Meconial } & \multicolumn{2}{c}{ Sanguinolento } \\
\cline { 2 - 7 } (Años) & $\mathbf{N}$ & $\%$ & $\mathbf{N}$ & $\%$ & $\mathbf{N}$ & $\%$ \\
\hline $35-39$ & 153 & $48,0 \%$ & 31 & $9,8 \%$ & 5 & $2,0 \%$ \\
\hline $40-44$ & 81 & $26,0 \%$ & 21 & $6,6 \%$ & 2 & $0,7 \%$ \\
$45-49$ & 18 & $6,0 \%$ & 2 & $0,6 \%$ & 1 & $0,3 \%$ \\
Total & $\mathbf{2 5 2}$ & $\mathbf{8 0 , 0} \%$ & $\mathbf{5 4}$ & $\mathbf{1 7 , 0 \%}$ & $\mathbf{8}$ & $\mathbf{3 , 0} \%$ \\
\hline
\end{tabular}

Según la tabla 8 se aprecia como hallazgo la presencia de líquido amniótico meconial en $17 \%$ de casos, sobre todo en el grupo etario entre 35-39 años de edad.

Tabla 9. Presencia de circular de cordón nucal según edad materna avanzada. Instituto Nacional Materno Perinatal. Enero-junio 2016.

\begin{tabular}{|c|c|c|c|c|c|c|}
\hline \multirow{3}{*}{$\begin{array}{c}\text { Edad } \\
\text { materna } \\
\text { avanzada } \\
\text { (años) }\end{array}$} & \multicolumn{6}{|c|}{ Presencia de circular de cordón nucal } \\
\hline & \multicolumn{2}{|c|}{1 Circular } & \multicolumn{2}{|c|}{2 circular } & \multicolumn{2}{|c|}{ Ninguna } \\
\hline & $\mathbf{N}$ & $\%$ & $\mathbf{N}$ & $\%$ & $\mathbf{N}$ & $\%$ \\
\hline $35-39$ & 29 & $9,2 \%$ & 6 & $2,0 \%$ & 158 & $49,6 \%$ \\
\hline $40-44$ & 14 & $4,5 \%$ & 6 & $2,0 \%$ & 84 & $26,4 \%$ \\
\hline $45-49$ & 1 & $0,3 \%$ & 0 & $0,0 \%$ & 19 & $6,0 \%$ \\
\hline Total & 44 & $14,0 \%$ & 12 & $4,0 \%$ & 261 & $82,0 \%$ \\
\hline
\end{tabular}

La presencia de circular de cordón nucal fetal se encontró en el $18 \%$ de casos; de ellos, el $4 \%$ tuvieron doble circular de cordón nucal y el $14 \%$ circular simple al cuello fetal (tabla 9). 
Tabla 10. Malformación congénita según edad materna avanzada. Instituto Nacional Materno Perinatal. Enerojunio 2016.

\begin{tabular}{ccccc}
\hline \multirow{2}{*}{$\begin{array}{c}\text { Edad materna } \\
\text { avanzada }\end{array}$} & \multicolumn{3}{c}{ Malformación congénita } \\
\cline { 2 - 5 } & $\mathbf{N}$ & $\mathbf{3}$ & $\mathbf{N}$ & No \\
\hline 35 - 39 años & 6 & $1,9 \%$ & 191 & $59,5 \%$ \\
\hline 40 - 44 años & 5 & $1,5 \%$ & 98 & $30,5 \%$ \\
\hline 45 - 49 años & 1 & $0,3 \%$ & 20 & $6,3 \%$ \\
\hline Total & $\mathbf{1 2}$ & $\mathbf{3 , 7 \%}$ & $\mathbf{3 0 9}$ & $\mathbf{9 6 , 0} \%$ \\
\hline
\end{tabular}

En la tabla 10 se aprecia que se encontró presencia de malformación congénita en el 3,7\% y de ellos la mitad de los casos corresponden al grupo etario comprendido entre 40-49 años de edad.

Tabla 11. Tipo de malformaciones congénitas según edad materna avanzada. Instituto Nacional Materno Perinatal. Enero-junio 2016.

\begin{tabular}{lccc}
\hline \multicolumn{1}{c}{ Malformación congénita } & \multicolumn{3}{c}{$\begin{array}{c}\text { Edad materna } \\
\text { avanzada (años) }\end{array}$} \\
\cline { 2 - 5 } & $\mathbf{3 5 - 3 9}$ & $\mathbf{4 0 - 4 4}$ & $\mathbf{4 5 - 4 9}$ \\
\hline Agenesia Renal + Encefalocele occipital & 1 & - & - \\
\hline Atresia duodenal & 1 & - & - \\
\hline $\begin{array}{l}\text { Atresia duodenal +cardiopatía } \\
\text { Enfermedad poliquística renal+Hipoplasia } \\
\text { pulmonar }\end{array}$ & - & 1 & - \\
$\begin{array}{l}\text { Hidrops fetal + Cardiopatía -+Hidrotórax } \\
\text { fetal }\end{array}$ & - & - & - \\
\hline Macrocefalia + Hidrocefalia & 1 & - & - \\
$\begin{array}{l}\text { Malformaciones fetales múltiples + } \\
\text { Hidrotórax fetal }\end{array}$ & 1 & - & - \\
\hline $\begin{array}{l}\text { Múltiples malformaciones craneofaciales } \\
\text { Onfalocele }\end{array}$ & 1 & - & - \\
\hline Síndrome de Down & 1 & - & - \\
\hline \multicolumn{1}{c}{ Total } & - & 3 & - \\
\hline
\end{tabular}

En la tabla 11 se aprecia que las principales malformaciones congénitas únicas encontradas como síndrome de Down están en el grupo etario comprendido entre 40-44 años de edad.

\section{DISCUSIÓN}

La morbilidad obstétrica y perinatal continúan siendo un problema de salud pública, en especial en gestantes en edad materna avanzada ${ }^{15,16}$, las cuales concuerdan con recientes investigaciones de autores como Hurtado CJ ${ }^{17}$ y Gutiérrez EC ${ }^{18}$ quienes reportan que en las edades extremas de la vida se presentan altas tasas de complicaciones obstétricas y perinatales.

Según Nolasco-Blé $\mathrm{AK}^{19}$ la vía más frecuente de terminación del embarazo fue la cesárea y la principal causa de indicación de cesárea es arbitrariamente el antecedente de cesárea; asimismo, estudios de Lemor $A^{20}$ reportan que la edad materna avanzada se asoció con una mayor tasa de cesáreas y más riesgo de tener un hijo con síndrome de Down; Ventura W y $\mathrm{col}^{21}$ reporta alta tasa de cesáreas $(56,8 \%)$ y mayor en el grupo de nulíparas (71,7\%); Baranda-Nájera $\mathrm{N}$ y col${ }^{22}$ reportan prevalencia de cesárea en $61,18 \%$; todas ellas concuerdan con nuestra casuística donde la vía de culminación del parto predominante fue la cesárea en $56 \%$ de los casos.

En nuestra casuística los principales hallazgos perinatales fueron: recién nacido con bajo peso al nacer (BPN) en $15 \%$ de la casuística sobre todo en el grupo etario de $40-$ 49 años de edad; presencia de líquido meconial(17\%); presencia de circular de cordón nucal fetal en $18 \%$; macrosomía fetal $(10 \%)$ y malformación congénita en $3,7 \%$ principalmente Sindrome de Down en el grupo etario de 40-44 años de edad; las que concuerdan con las publicaciones de Gutierrez EC ${ }^{18}$ que reportan complicaciones perinatales como distocia funicular $9.3 \%$, macrosomía fetal $7.6 \%$, distocia fetal $6 \%$ y prematuridad en $3,3 \%$.

Se puede concluir que en EMA los resultados perinatales frecuentes fueron bajo peso al nacer, presencia de líquido amniótico meconial, circular de cordón nucal fetal, macrosomía fetal y malformación congénita principalmente Sindrome de Down.

Financiamiento: autofinanciado.

Conflicto de interés: los autores declaran no tener algún conflicto de interés.

\section{REFERENCIAS BIBLIOGRÁFICAS}

1. Huang L, Sauve R, Birkett N, Fergusson D, van Walraven C. Maternal age and risk of stillbirth: a systematic review. CMAJ 2008; 178 (2): 165-72.

2. Ataulla I. The older obstetric patient. Current Obstet Gynaecol. 2005;15:46-53.

3. Hamilton BE, Martin JA, Ventura SJ. Births: preliminary data from 2007. Natl Vital Stat Rep 2009; 57:1-23.

4. Başer E, Seçkin KD, Erkılınç S, Karslı MF, Yeral IM, Kaymak $\mathrm{O}$, Çağlar T, Danışman N. The impact of parity on perinatal outcomes in pregnancies complicated by advanced maternal age. J Turkish-German Gynecol Assoc 2013; 14: 205-9.

5. Tipiani-Rodríguez O. ¿Es la edad materna avanzada un Factor de riesgo independiente para Complicaciones maternoperinatales?. Rev Per Ginecol Obstet. 2006;52(3):89-99

6. Baranda-Nájera N, Patiño-Rodríguez DM, Ramírez-Montiel ML, Rosales-Lucio J, Martínez-Zúñiga M,Prado-Alcázar J, Contreras-Carreto NA. Edad materna avanzada y morbilidad obstétrica. Evid Med Invest Salud 2014; 7 (3): 110-113

7. Curiel-Balsera E, Prieto-Palomino MA, Muñoz Bono J, Ruiz E, Galeas JL, Quesada García G. Análisis de la morbimortalidad materna de las pacientes con preeclampsia grave, eclampsia 
y síndrome de HELLP que Ingresan a la Unidad de Cuidados Intensivos Ginecoobstétrica. Med Intensiva. 2011; 35 (8): 478-483.

8. Lamminpaa R, Vehvilainen-Julkunen K, Gissler M, Heinonen S. Preeclampsia complicated by advanced maternal age: a registrybased study on primiparous women in Finland 19972008. BMC Pregnancy and Childbirth. 2012; 12: 47-50.

9. Flores Le-Roux JA, Benaiges Boix D, Botet JP. Diabetes mellitus gestacional: importancia del control glucémico intraparto. Clin Invest Arterioscl. 2012; 11: 1-

10. Bayrampour $\mathrm{H}$, Heaman M. Advanced maternal age and the risk of cesarean birth: A systema $\square$ c review. Birth. 2010; 37: 3-9.

11. Schoen $C$, Rosen T. Maternal and perinatal risks for women over 44. A review. Maturitas. 2009; 64 (2): 109-113.

12. Marín IR, Pérez G, Álvarez N. Hipertensión arterial y embarazo. Nefro plus. 2011; 4 (2): 21-30.

13. Vambergue A, Fajardy I. Consequences of gesta $\square$ onal and pregesta cional diabetes on placental func $\square$ on and birth weight. World J Diabetes. 2011; 2 (11): 196-203.

14. Instituto Nacional Materno Perinatal. Boletín estadístico periodo 2016. Lima Perú. Disponible en: http://www.inmp. gob.pe/institucional/boletines-estadisticos/1422371837.

15. Wang Y, Tanbo T, Abyholm T, Henriksen T. The impact of advanced maternal age and parity on obstetric and perinatal outcomes in singleton gestations. Arch Gynecol Obstet 2011; 284:31-37.

16. Chan BC, Lao TT. Effect of parity and advanced maternal age on obstetric outcome. Int J Gynaecol Obstet 2008;102:237241.

17. Hurtado CJ. Complicaciones obstetricas y perinatales en gestantes añosas y adolescentes atendidas en el hospital
Iquitos César Garayar García enero a diciembre 2013. (Tesis de pregrado). Lima, Perú: Facultad de Medicina Humana "Rafael Donayre Rojas" .Universidad de Amazonía Peruana, 2014. 48pp.

18. Gutierrez EC. Complicaciones obstétricas y perinatales en gestantes mayores de 35 años en el Hospital Rezola-Cañete en el año 2014. Tesis de Pregrado. Lima, Peru: Facultad de Obstetricia y Enfermería. Universidad San Martín de Porres, 2015. 46 pp. [Fecha de acceso 12 de enero del 2017] URL Disponible en: http://www.repositorioacademico.usmp.edu. pe/bitstream/usmp/2158/3/gutierrez_e.pdf [Fecha de acceso 12 de enero del 2017] URL Disponible en: http://dspace. unapiquitos.edu.pe/bitstream/unapiquitos/147/1/Tesis $\% 20$ Parte\%201.pdf.

19. Nolasco-Blé AK, Hernández-Herrera RJ, Ramos-González RM. Hallazgos perinatales de embarazos en edad materna avanzada. Ginecol Obstet Mex 2012;80(4):270-275

20. Lemor A. Relación entre la edad materna avanzada y la morbilidad neonatal en el Hospital Nacional Arzobispo Loayza. Enero -Octubre del 2012. Horiz Med 2014; 14(2): 22-26)

21. Ventura W y col. Embarazo después de los 40 años: características epidemiológicas. Rev Per Ginecol Obstel 2005;5(1):49-52

22. Baranda-Nájera N, Patiño-Rodríguez $D$, Ramírez-Montiel ML, Rosales-Lucio J, Martínez-Zúñiga M, Prado-Alcázar JJ, Contreras-Carreto NA. Edad materna avanzada y morbilidad obstétrica. Evid Med Invest Salud 2014; 7 (3): 110-113.

\section{Correspondencia:}

Dirección: Jirón Maracaibo 2153- San Martín de Porres-Lima 31. Teléfono: 567-6501, Celular: 999227657

E-mail: fayala1401@hotmail.com
ORCID iDs

Antonio Luna Figueroa

Félix Ayala Peralta

Enrique Guevara Ríos https://orcid.org/0000-0003-1527-8649

htps://orcid.org/0000-0002-2830-3789

(i) https://orcid.org/0000-0002-6962-2639 